\title{
Occurrence and characterization of Trichomitus batrachorum (Protista, Trichomonadidae) from Rhinella marina (Amphibia, Bufonidae) in the Philippines
}

\author{
Ianne Keziah O. Agripo ${ }^{1}$, Davin Edric V. Adao ${ }^{1}$ and Windell L. Rivera ${ }^{\text {* }}$
}

\begin{abstract}
Trichomonad isolation from amphibians is new in the Philippines as trichomonad studies in the country are few, limited only to mammals, reptiles and birds. Moreover, there are very few studies on amphibian-associated trichomonad ultrastructure and morphology. Trichomitus batrachorum (Ts. batrachorum) was isolated from Rhinella marina fecal samples and identified using SEM and TEM for ultrastructural study and 18S rRNA gene sequencing. A 37.5\% prevalence of $T$ s. batrachorum from $R$. marina was observed based on in vitro culture and molecular analysis. Characteristics of this coprozoic trichomonad that provided distinctive features for classification included body size and shape, three anterior flagella and a recurrent flagellum, lamelliform undulating membrane, type A costa periodicity, Vshaped parabasal body, well-developed pelta, shape and location of organelles such as the nucleus, blepharoplast, axostyle, comb-like organelle, hydrogenosomes and the observation of a pseudocyst stage. DNA sequence analysis corroborated these results, and generated phylogenetic trees with high bootstrap support further proved the identity of the isolate. The few identified trichomonads in the Philippines exhibit the capability for adaptation to new hosts and it is possible they have zoonotic potential. These findings contribute to the existing trichomonad data sets in the country. This is the first ultrastructural study of $T_{s}$. batrachorum species isolated from a toad.
\end{abstract}

Keywords: 18S rRNA gene, electron microscopy, Rhinella marina, Trichomitus batrachorum, ultrastructure

\section{Introduction}

Three trichomonad species are known to occur in frogs and toads: Trichomonas batrachorum Perty (Delvinquier and Freeland 1988; Dobell 1909), Trichomastix batrachorum Dobell, and Tritrichomonas augusta (Alexeieff) (Bozner 1996). Trichomonas batrachorum, Tritrichomonas batrachorum (Perty), and Trichomonas natricis Coutelen, Biguet \& Cochet are synonyms of Trichomitus batrachorum (Perty) (Frank 1984). Many trichomonad species belong to the Trichomitus batrachorum (Ts. batrachorum) complex due to morphological similarities and unknown physiological differences (Honigberg 1953). It can exist in trophozoite (Honigberg et al. 1972), pseudocyst (Mattern et al. 1973) and cyst (Brugerolle 1973).

\footnotetext{
${ }^{1}$ Institute of Biology, College of Science, University of the Philippines, Diliman, Quezon City 1101, Philippines

*Corresponding email: wlrivera@science.upd.edu.ph

Date Submitted: 08 May 2019

Date Accepted: 01 May 2020
}

Pseudocysts of $T s$. batrachorum have never been observed in feces, only in in vitro studies (Gillespie and Pearson 2001). Previous accounts showed the morphology through surface structural characterization of Ts. batrachorum from Bufo bufo (Germot et al. 1996), internal structural characterization of $T s$. batrachorum in trophozoite and pseudocyst stages from an American Type Culture Collection (ATCC) strain (Honigberg et al. 1972; Mattern et al. 1973), and its cyst stage through transmission electron microscopy (TEM) (Brugerolle 1973). Staining techniques using Schaudinn's fluid as fixative and ironalum haematoxylin as stain were commonly employed to observe cellular division and identification of these amphibian trichomonads (Delvinquier and Freeland 1988; Dobell 1909). In Rhinella marina (L.) toads from Australia, the presence of the trichomonads, Ts. batrachorum and Monocercomonas batrachorum (Dobell) have been microscopically identified through staining (Delvinquier and Freeland 1988). Ts. batrachorum have been isolated in the gut of reptiles (Frank 1984; Honigberg 1953; Whittington 1951). Identification of Ts. batrachorum in pigs from the Philippines using 18S rRNA gene sequence analysis implicates the potential of amphibian 
trichomonads to adapt to mammalian hosts (Dimasuay et al. 2013). In another study, Ts. batrachorum was isolated from an asymptomatic pig that was screened for intestinal trichomonads (Mostegl et al. 2012). Ts. batrachorum showed some similarities with Trichomonas vaginalis (Donné) and Tritrichomonas foetus (Riedmueller) in their proteolytic activities; however, the proteinase band patterns of $T$. vaginalis and Tt. foetus were more similar to each other compared to $T s$. batrachorum. These in turn may reflect differences in pathogenicity and habitat (Lockwood et al. 1984).

Trichomonads have been studied using light microscopy for prevalence, electron microscopy for morphological characterization, polymerase chain reaction and DNA sequencing for detection and identification of genetic diversity (Bellanger et al. 2008; Benchimol 2004; Cepicka et al. 2010; Gookin et al. 2007; Li et al. 2016). Microscopy of fecal/cloacal swabs are usually performed together with molecular tools to improve sensitivity of detection (Dimasuay and Rivera 2013; Kim et al. 2010; Li et al. 2016; Wartoń and Honigberg 1979). Both morphological characteristics and $18 \mathrm{~S}$ ribosomal RNA gene sequence data are being used in classification of parabasalids. Ultrastructural study using electron microscopy in combination with $18 \mathrm{~S}$ rRNA gene sequencing have been used to identify Tetratrichomonas buttreyi Hibler, Hammond, Caskey, Johnson \& Fitzgerald in a pig sample in a previous Philippine study (Rivera et al. 2008). These types of studies on field samples are especially important since biochemical or molecular studies are available only for a few axenic cultures (Kamaruddin et al. 2014; Malik et al. 2011). For the morphological characterization, interesting observations of details of surface structures of trichomonads could be anticipated using SEM. Trichomonads have structural features such as flagella and a well-developed undulating membrane which render them especially suitable for SEM analysis (Jensen and Hammond 1964; Wartoń and Honigberg 1979). The use of TEM has proven to be a reliable classification scheme for trichomonads since it provides additional characters for discerning evolutionary relationships which include the type of costa, presence of comb-like organelle, blepharoplast (indicates number of kinetosomes and respective flagella count) and peltar -axostylar complex (Benchimol 2004; Honigberg et al. 1972), and even the ability to form pseudocysts (Mattern et al. 1973).

Rhinella marina is a very abundant species of toad that has been put to various uses across its geographic range. It is an introduced species that is invasive. It is used for educational purposes (Solís et al. 2009) such as laboratory dissections in biology classes. $R$. marina was originally introduced to various countries to control populations of various insect pests of valuable crops such as sugarcane, but the cane toads soon became pests themselves by outcompeting or consuming local fauna (Global Invasive Species Database 2005). Observation of an uncharacterized and unidentified trichomonad species in an amphibian in the Philippines is new with respective classification and in-detail characterization of its morphology through SEM and TEM analyses. There is not much information on trichomonads from animals in the Philippines despite their increasing medical and veterinary importance. Therefore, the objective of this study was to identify trichomonad species in $R$. marina through the investigation of the genotypic, morphological, and ultrastructural characteristics using phylogenetic analysis, SEM, and TEM, respectively.

\section{Materials and Methods}

\section{Isolation and cultivation of trichomonads}

Fifty-six (56) fecal samples were collected from $R$. marina toads from various areas in Diliman, Quezon City, Philippines. These samples were inoculated in a biphasic medium containing agar slant overlaid with broth that is supplemented with $10 \%$ horse serum and antibiotics (1X penicillin and $0.1 \mathrm{~g} / \mathrm{ml}$ streptomycin) and incubated at room temperature for 3-5 d (Rivera 2008). Light microscopy was performed to detect motile trichomonads. Subculture was done four times or more before DNA extraction. In order to achieve a culture free from other protozoal contaminants, cultures were transferred to broth in 1.5 $\mathrm{mL}$ microfuge tubes at room temperature then subcultured back and forth to the biphasic medium until significant isolation of the trichomonads was achieved as confirmed by light microscopy.

Samples of the culture were submitted for SEM analysis to the Electron Microscopy Service Laboratory, National Institute of Molecular Biology and Biotechnology, University of the Philippines Los Baños, Laguna for further surface features characterization; and submitted for TEM analysis to the Electron Microscopy Laboratory, Research and Biotechnology Division, St. Luke's Medical Center, Quezon City, Philippines for internal ultrastructural study.

\section{Characterization through electron microscopy}

Prior to electron microcopy (EM) analyses, samples were washed thrice with $1 \mathrm{~mL}$ phosphate-buffered saline (PBS) with respective $10,000 \mathrm{rpm}$ centrifugation every after wash. The trichomonad samples for SEM were placed in $1.5 \mathrm{~mL}$ microfuge tubes with broth. The trichomonad samples for TEM were fixed in $5 \% \mathrm{w} / \mathrm{v}$ glutaraldehyde in $0.1 \mathrm{M}$ sodium cacodylate and were transported to the EM facility under cold condition.

For SEM, the trichomonads in broth were allowed to adhere into Thermonox coverslip at $4{ }^{\circ} \mathrm{C}$ and were fixed with 
$2.5 \%$ glutaraldehyde. After several washes with cacodylate buffer, the cells were post-fixed in $1 \%$ osmium tetroxide $\left(\mathrm{OsO}_{4}\right)$ at $4{ }^{\circ} \mathrm{C}$, dehydrated in increasing concentrations of ethanol, and then dried by hexamethyldisilazane (HMDS). The cells were kept in desiccator at room temperature, mounted on aluminum stub, and were examined under low vacuum mode using Phenom XL Table Top SEM.

The fixed sample for TEM analysis was washed with cacodylate buffer and subjected to centrifugation at 10,000 rpm for 5 min. Samples were secondarily fixed with $\mathrm{OsO}_{4}$ and stored in dark condition, washed with cacodylate buffer, dehydrated in increasing concentrations of acetone and then washed with $100 \%$ absolute acetone. A 1:3 ratio of acetone and media (chemical mixture) was added to the sample with respective mixing, centrifugation and decantation. Media was added and was placed in the rotator for $1 \mathrm{~h}$. The sample in the $1.5 \mathrm{~mL}$ microfuge tube was then transferred to a capsule beam, centrifuged and left to polymerize overnight at $70^{\circ} \mathrm{C}$. In order to produce sections to be placed in copper grids, the sample was sliced using the ultra-microtome and was sequentially stained with uranyl acetate and lead citrate. Copper grids with the sample were examined using Tecnai T12 TEM.

\section{DNA extraction}

Chelex DNA extraction was performed according to the procedure of Ong and Rivera (2010). Briefly, Ts. batrachorum cells from xenic cultures were harvested by centrifugation at $10,000 \mathrm{rpm}$ for $2 \mathrm{~min}$. The pellets were washed thrice by resuspension in $1 \mathrm{~mL}$ PBS and centrifugation at 10,000 rpm for 2 min. These were then suspended in $200 \mu \mathrm{L}$ of $5 \%$ Chelex (Sigma-Aldrich, St. Louis, MO) in sterile PCR-grade water followed by incubation at $56{ }^{\circ} \mathrm{C}$ for $30 \mathrm{~min}$. The tubes were vortexed for $10 \mathrm{~s}$ and incubated for $8 \mathrm{~min}$ at $100{ }^{\circ} \mathrm{C}$. DNA suspended in the supernatant was separated from the Chelex beads by centrifugation at $13,000 \mathrm{rpm}$ for $2 \mathrm{~min}$. The supernatant was stored at $-20^{\circ} \mathrm{C}$ in a new tube until use.

\section{PCR amplification, purification, and sequencing}

The T18 primer sets from the protocol of Dimasuay and Rivera (2013) were used in this study. These are T18SF (5'GGAAGCACACTTCGGTCATAG-3'), T18SRi (5'-CCTTCC GTCAATTCCTTCAA-3'), T18SFi (5'-GTCGATCAAGGGCG AGAGTA-3'), and T18SR (5'-CGTTACCTTGTTACGACT TCTCC-3'). The primer pair T18SF/T18SRi amplifies the first $900 \mathrm{bp}$ of the $18 \mathrm{~S}$ rRNA gene sequence while the primer pair T18SFi/T18SR amplifies the remaining 800 bp. PCR amplification was carried out in duplicate using Promega PCR Master Mix (Promega Corporation, Wisconsin, USA) along with negative and positive controls. PCR products were visualized in $2 \%$ agarose gel containing Gel Red under UV light and compared to the 1000-bp molecular weight ladder (KAPPA Universal Ladder). PCR products were sent to Macrogen, Inc. (South Korea) for purification and direct sequencing.

\section{Phylogenetic analyses}

The generated sequences were assembled, concatenated and aligned using CLUSTAL $\mathrm{W}$ algorithm in BioEdit 7.2.5 software (Hall 1999) and later uploaded onto BLAST NCBI (http://blast.ncbi.nlm.nih.gov/BLAST.cgi) to identify similar sequences in known data sets. The optimal model for DNA substitution was determined through jmodeltest feature in MEGA7 (Kumar et al. 2016). The same program was used to construct Neighbor-Joining (NJ), Maximum Parsimony (MP), and Maximum-Likelihood (ML) phylogenetic trees with 1000 bootstrap replicates. Clusters in the phylogenetic trees were considered valid if bootstrap support were greater than $50 \%$ (out of 1000 bootstraps) for NJ, ML and MP. All trees were rooted to Trichonympha agilis Leidy (GU461590).

\section{Nucleotide sequence accession numbers}

The nucleotide sequences reported in this paper were deposited in GenBank under accession numbers, MH321549 MH321574.

\section{Results and Discussion}

\section{Summary of trichomonad isolates}

A total of 56 toad fecal samples were obtained and 28 $(50 \%)$ of these had motile trichomonads. Seven (7) positive isolates were not used for phylogenetic analysis due to negative PCR results $(\mathrm{n}=2)$ and mixed sequences $(\mathrm{n}=5)$. Thus, the prevalence of $T s$. batrachorum was $37.5 \%(21 / 56)$ from $R$. marina based on both in vitro culture and molecular analysis.

\section{Light and electron microscopy}

Cultures of trichomonads free from other protozoa generally survived for at least three weeks in the biphasic medium and in broth medium. The cultures grew well at room temperature although microbial contaminants were still present in all cultures grown in biphasic medium. Smaller sizes of putatively identified trichomonads were also observed because of their similarity in locomotion and shape. Under light microscopy, trichomonads showed ovoid shape but with pointed posterior tip that coalesces at its axostyle which was minimally observed. However, the number of flagella and undulating membrane could not be discerned using a light microscope. Locomotion can be best described as irregular, spasmodic and fast, and oftentimes, the trichomonads move about in a wave- 
Table 1. BLAST results of the 26 trichomonad isolates from Rhinella marina stool samples. The table indicates a 94-96\% similarity of trichomonad isolates to Trichomitus batrachorum (JX565060.1) and Trichomitus batrachorum (AF124610.1) with a $97-100 \%$ query cover.

\begin{tabular}{|c|c|c|c|}
\hline \multirow{2}{*}{ Isolate (26) } & \multicolumn{3}{|c|}{ BLAST Result } \\
\hline & Species Identity & $\%$ Species Similarity & $\%$ Query Cover \\
\hline IB2: T. batrachorum (MH321549) & T. batrachorum (JX565060.1) & $96 \%(1424 / 1484)$ & $99 \%$ \\
\hline IB6: T. batrachorum (MH321550) & T. batrachorum (JX565060.1) & $96 \%(1427 / 1489)$ & $99 \%$ \\
\hline IB8: T. batrachorum (MH321551) & T. batrachorum (JX565060.1) & $96 \%(1428 / 1488)$ & $99 \%$ \\
\hline IB 9: T. batrachorum (MH321552) & T.batrachorum (JX565060.1) & $96 \%(1436 / 1497)$ & $98 \%$ \\
\hline IB 11: T. batrachorum (MH321553) & T. batrachorum (AF124610.1) & $96 \%(1389 / 1448)$ & $97 \%$ \\
\hline IB13: T. batrachorum (MH321554) & T.batrachorum (JX565060.1) & $96 \%(1423 / 1482)$ & $99 \%$ \\
\hline IB19: T. batrachorum (MH321555) & T.batrachorum (JX565060.1) & $96 \%(1428 / 1490)$ & $100 \%$ \\
\hline IB 20: T. batrachorum (MH321556) & T.batrachorum (JX565060.1) & $96 \%(1435 / 1495)$ & $99 \%$ \\
\hline IB23: T. batrachorum (MH321557) & T.batrachorum (JX565060.1) & $96 \%(1421 / 1487)$ & $99 \%$ \\
\hline IB 33: T. batrachorum (MH321558) & T.batrachorum (JX565060.1) & $95 \%(1425 / 1498)$ & $99 \%$ \\
\hline IB 34: T. batrachorum (MH321559) & T. batrachorum (AF124610.1) & $95 \%(1411 / 1478)$ & $99 \%$ \\
\hline QT 20: T. batrachorum (MH321560) & T.batrachorum (JX565060.1) & $96 \%(1433 / 1495)$ & $99 \%$ \\
\hline QT 29: T. batrachorum (MH321561) & T.batrachorum (JX565060.1) & $96 \%(1433 / 1494)$ & $99 \%$ \\
\hline QT 43: T. batrachorum (MH321562) & T. batrachorum (AF124610.1) & $96 \%(1430 / 1493)$ & $98 \%$ \\
\hline QT 51: T. batrachorum (MH321563) & T.batrachorum (JX565060.1) & $96 \%(1434 / 1496)$ & $99 \%$ \\
\hline QT 56: T. batrachorum (MH321564) & T. batrachorum (AF124610.1) & $96 \%(1428 / 1492)$ & $98 \%$ \\
\hline QT 58: T. batrachorum (MH321565) & T. batrachorum (AF124610.1) & $96 \%(1425 / 1485)$ & $99 \%$ \\
\hline QT 59: T. batrachorum (MH321566) & T.batrachorum (JX565060.1) & $94 \%(1414 / 1500)$ & $99 \%$ \\
\hline QT 60: T. batrachorum (MH321567) & T.batrachorum (JX565060.1) & $96 \%(1423 / 1487)$ & $99 \%$ \\
\hline QT 62: T. batrachorum (MH321568) & T.batrachorum (JX565060.1) & $96 \%(1435 / 1495)$ & $99 \%$ \\
\hline QT 63: T. batrachorum (MH321569) & T. batrachorum (AF124610.1) & $94 \%(1404 / 1497)$ & $99 \%$ \\
\hline QT 65: T. batrachorum (MH321570) & T.batrachorum (JX565060.1) & $94 \%(1428 / 1513)$ & $99 \%$ \\
\hline QT 67: T. batrachorum (MH321571) & T. batrachorum (AF124610.1) & $94 \%(1403 / 1495)$ & $99 \%$ \\
\hline QT 69: T. batrachorum (MH321572) & T.batrachorum (JX565060.1) & $96 \%(1431 / 1494)$ & $99 \%$ \\
\hline QT 71: T. batrachorum (MH321573) & T. batrachorum (AF124610.1) & $95 \%(1429 / 1499)$ & $99 \%$ \\
\hline QT 74: T. batrachorum (MH321574) & T.batrachorum (JX565060.1) & $96 \%(1426 / 1492)$ & $99 \%$ \\
\hline
\end{tabular}


like motion. Clumping of trichomonads in areas with debris was also prevalent. The general morphology of the trichomonad and the relevant structures associated with this protozoon are in line with previous studies (Benchimol 2004). The body of the trophozoite ranged in length from $7.8-15.1 \mu \mathrm{m}$ (average $=$ $9.78 \pm 2.2 \mu \mathrm{m}$ ) and in width from $3.2-4.98 \mu \mathrm{m}$ (average $=4.08$ $\pm 0.6 \mu \mathrm{m}$ ), while pseudocysts have $5.0-7.9 \mu \mathrm{m}$ (average $=6.33$ $\pm 1.02 \mu \mathrm{m})$ by $2.65-5.81 \mu \mathrm{m}(4.69 \pm 0.86 \mu \mathrm{m})$. The pyriformshaped trophozoite and its pseudocyst were both observed in SEM and TEM micrographs. The pelta-axostylar complex and the costa are the main cytoskeleton structures observed. The axostyle is a hyaline rod, which showed a flattened capitular expansion. The capitulum, next to the right ventro-lateral surface of the nucleus, is the wider anterior part of the axostyle extending from the peltar-axostylar junction to the posterior end of the nucleus as well as the axostylar trunk which forms a tube posteriorly from the nucleus (Fig. 1A). A row of accessory microtubules is found near the peltar-axostylar junction in longitudinal section appearing external to and almost perpendicular to the axostylar microtubules (Fig. 1A). The axostyle appears to narrow progressively until the tail-like terminal part protrudes from the cell posterior (Fig. 1B). Numerous glycogen granules along with some large vesicles and hydrogenosomes are present in the cytoplasm. Notably these glycogen granules also localize in the axostyle (Fig. 1B). The pelta, large crescent-shaped structure situated near the anterior surface, is a structure seen to be extending into the periflagellar canal wall and appears to support the canal from which the anterior flagella emerge (Fig. 1C, 1D).

The trichomonads possess an undulating membrane (Fig. $1 \mathrm{~B}, 2 \mathrm{~A})$ which is supported by a cytoskeletal structure, the costa (Fig. 2B), a large striated root fibril originating from the kinetosomal complex specifically from kinetosome \# 3 (Fig. $2 \mathrm{C}$ ). The undulating membrane is constituted by both the marginal lamellae and the recurrent flagellum (Fig. 2A). The former originates ventral from the junction of the recurrent flagellum and its kinetosome R (Fig. 2B). The accessory filament is a segment of the fold distal to the recurrent flagellum (Fig. 2A). The latter should extend beyond the membrane as a free flagellum. The costa is seen as a slender rod with an equal or greater diameter compared to the anterior flagella and it also includes a lateral arcuate system (LAS) (Fig. 2B, 2C). It consists of repeating four transverse bands considered to be of type A costa structure (Fig. 2D) similar to the Ts. batrachorum. An additional periodic structure, the comb-like organelle, which lies lateral to the costa is observed (Figs. 2B, 2C).

Three anterior flagella of unequal lengths originated from the periflagellar canal (Figs. 1C, 1D) and one recurrent flagellum were observed. The latter is connected to the main body of the
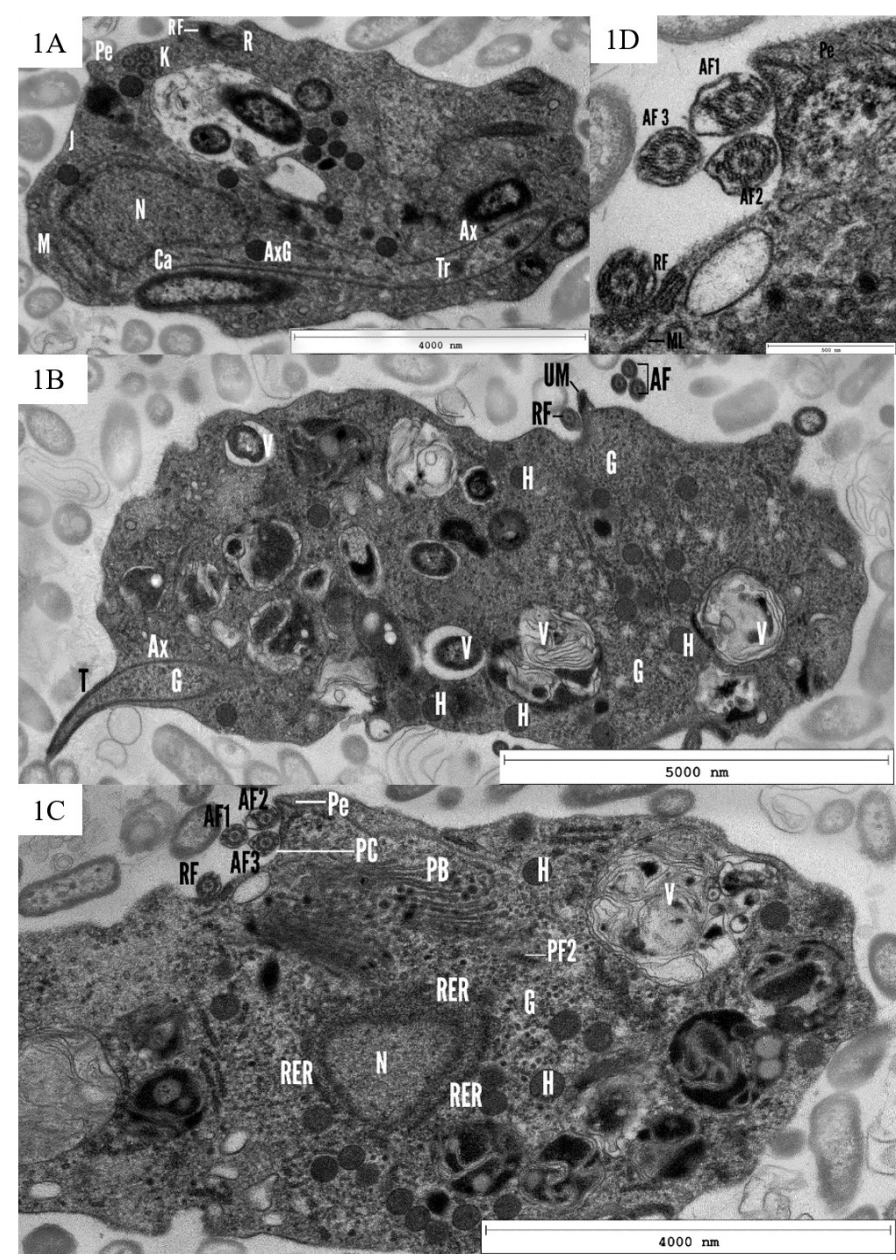

Figure 1. Ts. batrachorum trophozoite electron micrographs. A. The capitulum (Ca) is seen as the flat part of the axostyle (Ax) extending from the peltaraxostylar junction $(\mathrm{J})$ on the anterior end of the nucleus $(\mathrm{N})$ all the way to the posterior end of the latter structure. The trunk is the tubular segment posterior to the nucleus. Axostylar granules $(\mathrm{AxG})$ or hydrogenosomes are found within the axostyle. A row of accessory microtubules (M) are located near the peltaraxostylar junction $(\mathrm{J})$ which appear as striations external to and almost perpendicular to the axostylar microtubules. Also visible are the three kinetosomes $(\mathrm{K})$ anterior to the recurrent flagellum (RF), the kinetosome $\mathrm{R}(\mathrm{R})$, the Pelta (Pe), and the axostylar trunk (Tr). The oblique "parasaggital section" of the recurrent flagellum is minimally observed. Fig. 1 A X 4,400. B. The axostyle (Ax) and the tail-like terminal part $(\mathrm{T})$ are partially visible near the posterior end. Glycogen granules $(\mathrm{G})$, hydrogenosomes $(\mathrm{H})$ and large vesicles or food vacuoles $(\mathrm{V})$ are clearly presented within the cytoplasm. Cross-sections of the three anterior flagella (AF) and the recurrent flagellum (RF) are presented. A section of the undulating membrane (UM) is visible anterior to the RF. Fig. 1B X 3,200. C. The three anterior flagella (AF1, AF2, AF3) are seen in the periflagellar canal (PC) and the recurrent flagellum (RF). The pelta (Pe) appears in a section of the periflagellar canal (PC). A minimal portion of the parabasal filament 2 (PF2) is seen posterior and dorsal under the longitudinally sectioned parabasal body (PB). The nucleus $(\mathrm{N})$ is seen surrounded by the rough endoplasmic reticulum (RER) and the distributed glycogen granules $(\mathrm{G})$, vacuoles (V), and hydrogenosomes $(\mathrm{H})$ in the cytosol. Fig. 1C X 4,400. D. Thin section showing the " $9+2$ " arrangement of microtubules in the flagellar axonemes (AF1, AF2, AF3, RF). The marginal lamellae (ML) with observable periodicity and the pelta $(\mathrm{Pe})$ flanking the opening of the periflagellar canal are also seen. Fig. 1D X 30,000. 
cell forming the undulating membrane (Fig. 2A). The axonemes of these flagella have 9+2 arrangement of microtubules (Fig. 1D) and originates in the kinetosomal complex. The flagella originate from the blepharoplast area where the kinetosomal complex is situated (Figs. 1A, 2B, 2C, 2E). Kinetosome \#1, \#2, and $\# 3$ of the anterior flagella with their respective filaments are observed anteriorly of the body. The kinetosome R is minimally observed together with the basal body of the recurrent flagellum and also emerges in the anterior region but from a different opening (Figs. 1A, 1C, 1D) and its distal part is free and not part of the undulating membrane (Fig. 2F). The undulating membrane which runs the length of the cell, typically has $4-5$ bold undulations (Fig. 2F).

The Golgi complex or parabasal body is a microfibrillar strand linked to the kinetosomes. The parabasal body consists of peripherally vesiculated cisternae (Figs. 1C, 2C, 2E) together with two parabasal filaments (Fig. 1C). The rough endoplasmic reticulum (RER) in trichomonads surrounds the nucleus and forms the outer membrane of the nuclear envelope (Fig. 1C). It is also adjacent to the capitulum which in turn is adjacent to the surface posterior of the nucleus. There is close association of the RER with the axostyle and hydrogenosomes (Fig. 1C). The nucleus is usually broadly ellipsoidal or ovoidal. Other structures observed are hydrogenosomes which are spherical organelles usually associated with glycogen particles. The food vacuoles were big circular structures, almost the same size as the nucleus and it contained digested material (Fig. 1B). Glycogen granules were distributed throughout the cytoplasm of the trichomonad cell which are dark in color (Figs. 1B, 1C).

Some trichomonads formed cells with internalized flagella referred to as pseudocysts. Longitudinal section of the flagella inside the cell supports the observation of trichomonads under pseudocyst form (Figs. 3A, 3B). In this form, the flagella are internalized and are located in a canal with no direct contact with the cytoplasm (Fig. 3A). The recurrent flagellum and anterior flagella are situated in separate canals (Fig. 3B). The axial position and shape of the axostyle and costa change to a curved form giving the cell a more rounded shape (Figs. 3B, 3C, 3D). The final stages of flagellum internalization or the initial phases of the budding process of a pyriform cell from a pseudocyst are observed where the anterior flagella are already externalized (Fig. 3E).

The results of both SEM and TEM showed features similar to the ultrastructure of the trichomonad, Ts. batrachorum (Dobell 1909; Honigberg 1953; Honigberg et al., 1972; Mattern et al., 1973; Samuels 1957). Ts. batrachorum is a cosmopolitan species recorded in various amphibians in the world (Delvinquier and Freeland 1988). Observations through light microscopy were only limited to locomotion, body shape and

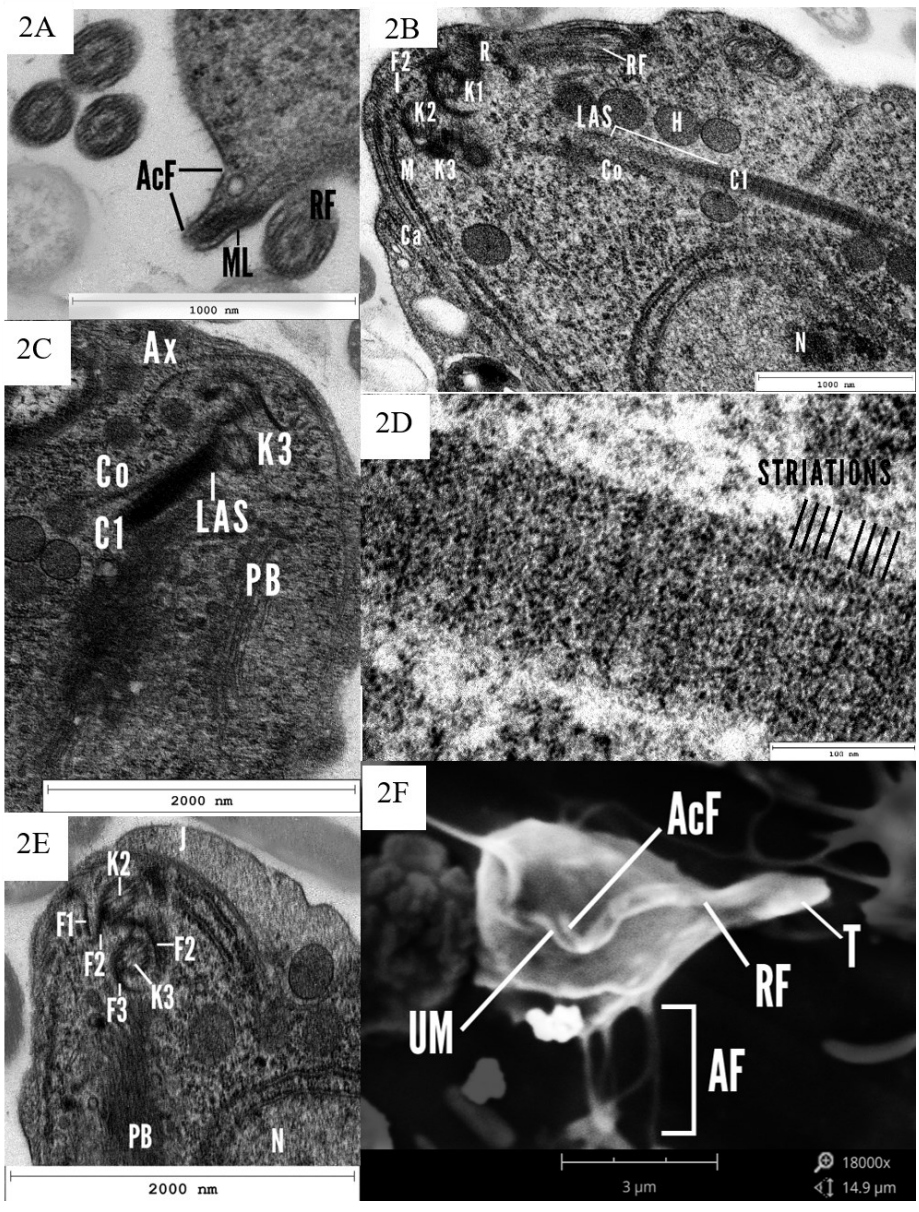

Figure 2. Ts. batrachorum trophozoite electron micrographs. A. Transverse section of the undulating membrane with the marginal lamellae (ML), accessory filament (AcF), and recurrent flagellum (ML) visible. Fig. 2A X 15,000. B. Kinetosome \#1 is seen together with Kinetosome \#2 with filaments (F2) visible on the inner surface of the pelta. The kinetosome $R(R)$ is minimally observed together with the basal body of the recurrent flagellum (RF). The section includes the costa $(\mathrm{C} 1)$ with the periodic comb-like organelle $(\mathrm{Co})$ and the lateral arcuate system (LAS). The row of microtubules (M) are external and closely opposed to those of the axostylar capitulum $(\mathrm{Ca})$. The nucleus $(\mathrm{N})$ is also present. Fig. 2B X 11,000. C. Clearly seen is the kinetosome \#3 (K3) as the origin of the costa $(\mathrm{C} 1)$ in the kinetosomal complex. The comb-like organelle (Co) and lateral arcuate system (LAS) supports the costa (C1). The cross section of the axostyle (Ax) and the parabasal body (PB) is also observed. Fig. 2C X 6,500 . D. A close view of the costa showing Type A structure which consists of the periodic repetition of four transverse bands. Fig. 2D X 110,000. E. Kinetosome \#1 (K1) and Kinetosome \#2 (K2) with their respective filaments (F1 and F2) are evidently observed. The peltar-axostylar junction ( $\mathrm{J}$ ) region is demarcated. Parabasal body (PB) and the nucleus (N) are minimally observed. Fig. 2E X 6,500. F. Surface structural morphology of $T$. batrachorum which is an observation of a pyriform cell. The free external margin of the undulating membrane (UM) consists of the attached segment of the recurrent flagellum $(\mathrm{RF})$ and the accessory filament $(\mathrm{AcF})$. The flagellum should continue as a free posterior flagellum (PF) for about one-third of its total length beyond the end of the undulating membrane (UM) but it is not seen in this view. The periflagellar canal cannot be seen and the same orientation of origin of the three anterior flagella is vaguely observed. Fig. 2F X 18,000. 
size which provided minimal information to detect differences. However, through the use of SEM, the number of flagella, the type of undulating membrane (UM), and the presence of both trophozoite and pseudocyt stages were observed. Through TEM, the body size and shape, costa periodicity, degree of pelta development, and the shape and location of organelles such as the nucleus, parabasal body, kinetosomal complex, axostyle, comb-like organelle and hydrogenosomes were observed. Primarily the Type A costa which is a periodic repetition of four transverse bands, well-developed pelta, lamelliform undulating membrane, four flagella and the comb-like structure suggested that the isolated trichomonad is part of the class Hypotrichomonadea and possibly belongs to the genus Trichomitus. Ultrastructure supports the position of Trichomitus in the evolutionary sequence, Monocercomonas to Hypotrichomonas to Trichomitus to the Trichomonadinae (Honigberg et al. 1972). Trichomitus also shares the comb-like structure with all Tritrichomonadinae, which is not found either in Hypotrichomonas or Trichomonadinae and the Type A periodicity (Honigberg et al. 1972). In the genus Trichomitus, the UM presents few undulations (Germot et al. 1996) and is slightly shorter than the body (Marinkelle 1968) which is in accordance to what was observed. Only Trichomitus has both the costal base and the comb-like organelle among genera of the type characteristic of the Trichomonadinae with a welldeveloped undulating membrane (Honigberg et al. 1972). The length of the flagella was difficult to measure, however, from the available SEM micrograph, it is more or less unequal. This is considered a restriction in isolate classification. The generated body size average for trophozoite and pseudocyst stages is quite variable and does not give much distinction. Hence, the stage and size may not be a good basis for classification since there is a doubt on the validity of length measurements in Ts. batrachorum for species designation (Samuels 1957).

A total of 26 samples full-length aligned 18S rRNA gene sequences with lengths of 1472 nucleotide positions were used for generating the respective phylogenetic tree and identified using BLAST. Additional five DNA samples were from previously stored DNA extracts from the same host (QT 20, QT 29, QT 43, QT 51, QT 56). A total of 22 samples were most similar to Ts. batrachorum with $95-96 \%$ sequence similarity and $97-100 \%$ coverage while 4 samples (QT59, QT63, QT65, QT67) were only 94\% similar to Ts. batrachorum with 99$100 \%$ coverage based on BLAST results (Table 1). All samples clustered with $T s$. batrachorum GenBank sequences with high bootstrap support but isolates QT63 and QT67 formed a separate cluster with the other Ts. batrachorum isolates in this study (Fig . 4). The consensus tree showed 6 clades supported by high bootstrap values (Fig. 4). The genera Pentatrichomonas, Trichomonas, and Tetratrichomonas compose a single clade belonging to the class Trichomonadea. The genera Monocercomonas and Tritrichomonas which belong to the class Tritrichomonadea formed its own clade. The class Hypotrichomonadea formed its own clade including the trichomonad isolates from the toads. The isolates clustered with the Ts. batrachorum-group with bootsrap support of 100/97/100 (ML/MP/NJ). The Hypotrichomonas acosta-group formed a sister clade to the $T s$. batrachorum-group with bootstrap support of 90/82/78 (ML/NJ/MP) within the class Hypotrichomonadea clade.

R. marina toads harbor Ts. batrachorum as shown in the BLAST results and phylogenetic tree. Phylogenetic analysis using the ML, MP, and NJ methods supported the data of the BLAST analysis. BLAST results showed $94-96 \%$ similarity. However, it should be noted that $95-99 \%$ similarity of $18 \mathrm{~S}$ rRNA gene sequence with reference strains is needed to show relatedness at the species or genus le vel (Stoeck and Epstein 2003). The $18 \mathrm{~S}$ rRNA gene sequence of samples QT 67 and QT 63 did not cluster with the rest of the Ts. batrachorum and were only $94 \%$ similar to $T$ s. batrachorum reference sequences (GenBank accession no. AF124610.1). This could suggest another Trichomitus species found only in cane toads in the Philippines, but more evidence is required. It is possible for other commensal trichomonads to thrive in one host apart from the dominant trichomonad commonly observed in the particular host which has been exhibited in mammals (Mostegl et al. 2012) and birds (Grabensteiner et al. 2010). The constructed phylogenetic tree in this study shows similar results with the new classification of Parabasalia (Cepicka et al. 2010) which supports the general clustering in the classes Trichomonadea, Hypotrichomonadea and Tritrichomonadea.

The few identified trichomonads in the Philippines exhibit the capability for adaptation to new hosts and apart from zoonotic transmission, pathogenesis in host is also possible (Dimasuay and Rivera 2013). The identification of amphibian trichomonads is important to further assess possible patterns of host range and risks of zoonotic transmission. The molecular characterization and pathogenicity of trichomonads are illunderstood because some species are conditionally pathogenic only and even the phylogenetic trees for Parabasalia are continually being updated (Cepicka et al. 2010). There are no studies yet that prove the pathogenicity of novel trichomonads isolated from frogs and toads (and other poikilothermic animals) (Frank 1984). Ts. batrachorum was previously observed from an albino snake and iguanas (Dimasuay and Rivera 2013) and from a pig (Dimasuay et al. 2013) in the Philippines. Mostegl et al. (2012) also reported the presence of Ts. batrachorum in pigs. 


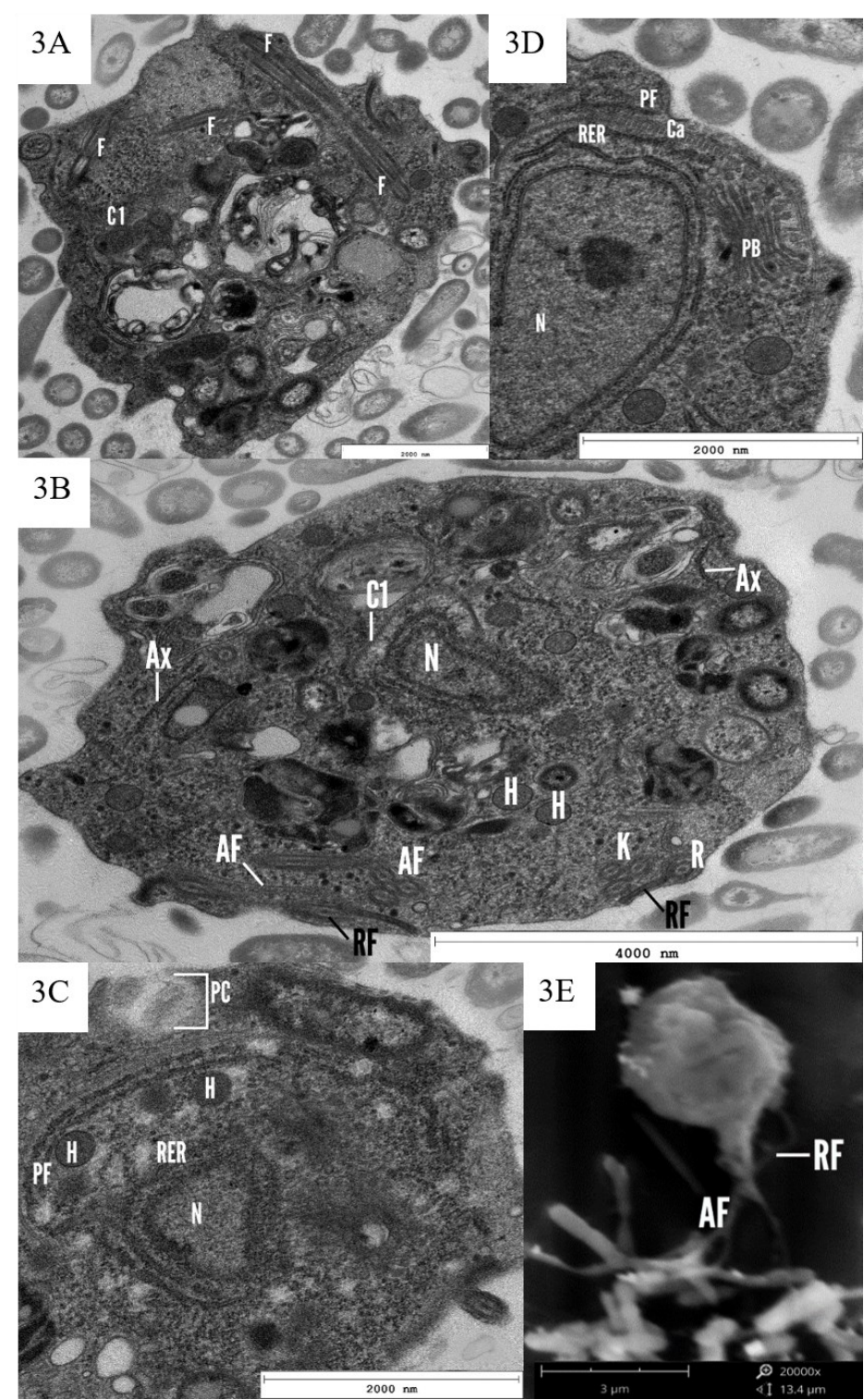

Figure 3. Ts. batrachorum pseudocyst electron micrographs. A. Globular pseudocyst stage showing the internalized flagella $(\mathrm{F})$ and the kinetosomes $(\mathrm{K})$. The deformed curved costa (C1) is also visible. Fig. 3A X 6,500. B. The curved axostyle (Ax) and Costa (C1) are observed. Internalized flagella are displayed and located in canals, noting that recurrent flagellum and anterior flagella have different canals and do not come in contact with the cytoplasm. Fig. 3B X 6,500. C. The rough endoplasmic reticulum (RER) is adjacent to the nucleus (N) which is in close association with some hydrogenosomes $(\mathrm{H})$. The parabasal filament (PF) courses throughout the body. The periflagellar canal (PC) is observed with the faintly seen anterior flagella. Fig. 3C X 6,500. D. The rough endoplasmic reticulum (RER) and $\backslash$ nucleus $(\mathrm{N})$ are found next to the capitulum (Ca). Adjacent to the posterior surface of the nucleus are parallel microtubules of the wide axostylar capitulum (Ca). The parabasal filament (PF) and the cisternae of the V-shaped parabasal body (PB) is seen. Fig. 3D X 6,500. E. SEM of a pseudocyst. The anterior flagella $(\mathrm{AF})$ and the recurrent flagellum (RF) are vaguely observed. Fig. 3E X 20,000.
The presence of $T s$. batrachorum in mammals, reptiles, and amphibians exhibits the extent of transfer and host range of this trichomonad. The mechanism of transfer from amphibian and reptile hosts to mammals still needs further study. Previous studies implicate an oral-fecal route of transmission through ingestion of trophozoites and cysts (Dimasuay and Rivera 2013; Marinkelle 1968).

R. marina, which is native to South America, exhibits its ability to retain its intestinal protozoan, $T$ s. batrachorum, despite introduction to other areas in the world (Delvinquier and Freeland 1988). Since the study of Dobell (1909), there have been extensive research on the ultrastructural characteristics of Ts. batrachorum, isolated from a rat or pilot snake. Honigberg et al. (1972) and Mattern et al. (1973) observed both trophozoite and pseudocyst stages using TEM. Isolation of Ts. batrachorum from B. marinus (syn. R. marina) has already been done by Delvinquier and Freeland (1988), however, observations were limited only to staining and no ultrastructural study was done. Ultrastructure of Ts. batrachorum in this study was similar to those from the studies of Honigberg et al. (1972) and Mattern et al. (1973) on ATCC isolates. This shows that Ts. batrachorum morphology and ultrastructure remain similar even if transferred to other hosts or to similar hosts but in other locations. However, it is still a possibility that there may be physiological differences.

Trichomonads typically have trophozoite and pseudocyst forms only (Brugerolle 1973; Farmer 1993) but Ts. batrachorum also exhibit a cyst stage form. Despite the observation of trophozoite and pseudocyst stages, its true cyst form was not observed. During pseudocyst formation, the shape of the parasite became spherical and all the flagella were internalized. $T$ s. batrachorum pseudocysts are presumed to form through dissociation of mastigont organelles into their component ultrastructural forms (Mattern et al. 1973). However, pseudocysts are still able to divide with their microtubular structures such as flagella and axostyle intact with the latter and the costa in a curved form giving the cell its round shape (Pereira-Neves et al. 2003). The flagella can be located in endocytic vacuoles or canals without direct contact with the cytoplasm (Granger et al. 2000). The pseudocysts have a smaller diameter compared to trophozoite due to its globular shape. Minimal information is known about the behavior and role of pseudocysts in the trichomonad cycle but because of its reversible formation, it represents a defense mechanism against unfavorable environmental conditions (Pereira-Neves et al. 2003). So far, pseudocysts have only been observed in in vitro studies (Gillespie and Pearson 2001). Pseudocyst formation was observed in this study during transport possibly because of stress such as $\mathrm{pH}$ alterations and nutrient depletion (Borges et al. 


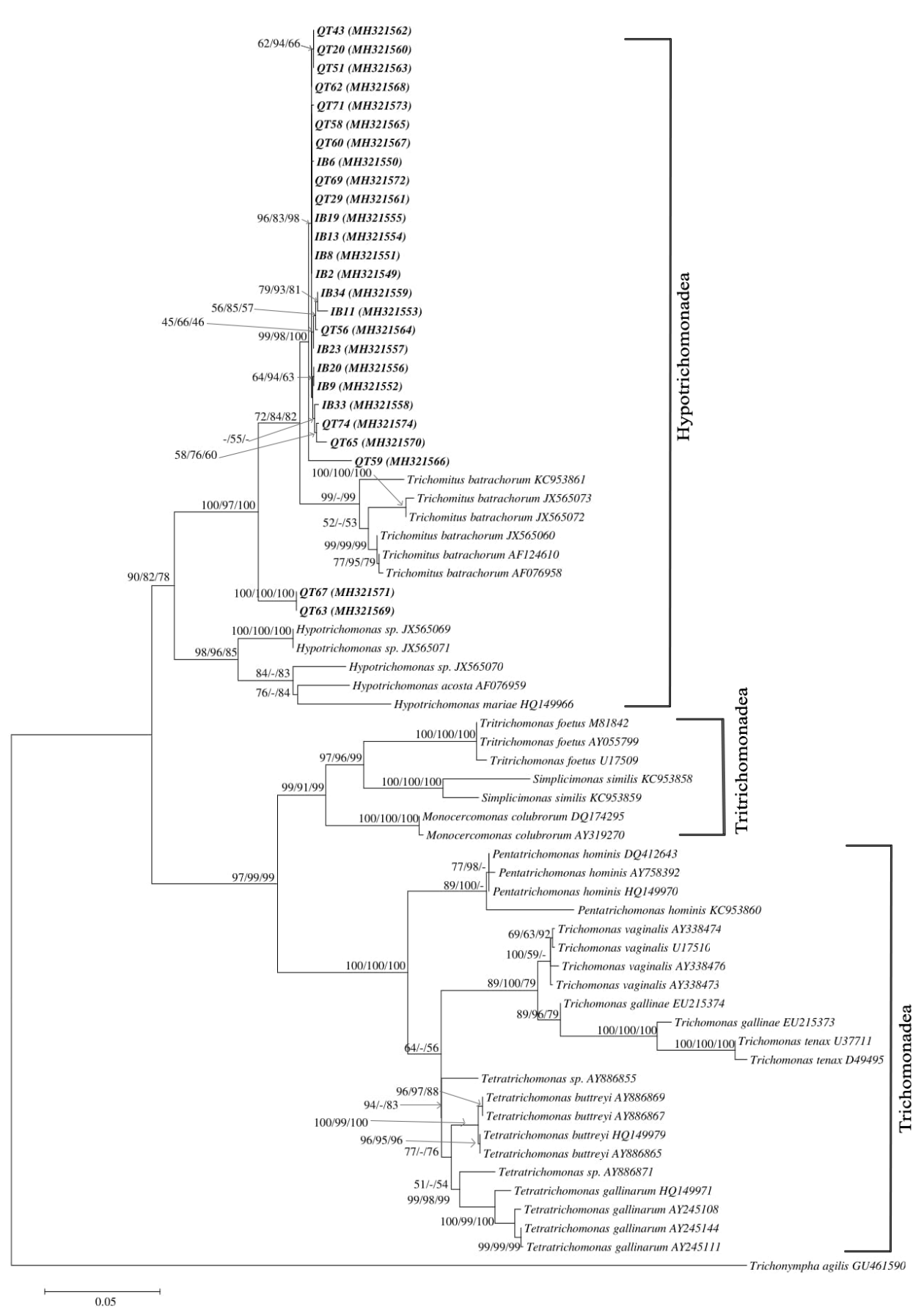

Figure 4. Phylogenetic tree of the Philippine and reference Ts. batrachorum isolates, based on the 18S rRNA gene (1633 bp). The Philippine isolate designation and GenBank numbers are shown in bold. The tree, constructed with ML analysis using GTR $+\mathrm{G}+\mathrm{I}$ as model of DNA substitution, was rooted with Trichonympha agilis. The three values at the nodes represent the bootstrap values from ML, MP, and NJ, respectively. Low bootstrap values $(<50 \%)$ are not shown. Scale bar indicates 5 nucleotide changes for every 100 nucleotides. 
2004) and lack of fresh medium necessary to revert to its trophozoite form (Pereira-Neves et al. 2003).

\section{Conclusions and Recommendations}

Ts. batrachorum was identified in R. marina stool samples based on ultrastructural and molecular analyses. Unlike the published articles that studied both fine structure and pseudocyst formation through TEM only, this study revealed the surface characterization of the trichomonad in both trophozoite and pseudocyst stages. In addition, this study obtained isolates through cultivation of fresh toad fecal samples unlike previous studies where Ts. batrachorum came from axenic ATCC cultures from a rat or pilot snake (Honigberg et al. 1972; Mattern et al. 1973). Identification of Ts. batrachorum in $R$. marina was done only through morphological characterization by staining and no DNA sequencing was done to support classification (Delvinquier and Freeland 1988). This is the first ultrastructural study of Ts. batrachorum species isolated from a toad. Future studies should focus on identification of other trichmonad species in toads as well as other amphibians and reptiles. Identification of trichomonad species in these hosts can show their full host range and possible links of zoonotic transmission.

\section{Acknowledgement}

This study was financially supported by the National Research Council of the Philippines of the Department of Science and Technology.

\section{Literature Cited}

Bellanger, A.P., O. Cabaret, J.M. Costa, F. Foulet, S. Bretagne, $\&$ F. Botterel, 2008. Two unusual occurrences of trichomoniasis: rapid species identification by PCR. Journal of Clinical Microbiology, 46: 3159-3161. DOI: 10.1128/JCM.00322-08

Benchimol, M., 2004. Trichomonads under microscopy. Microscopy and Microanalysis, 10: 528-550. DOI: 10.1017/S1431927604040905

Borges, F.P., R.C.M. Wiltuschnig, T. Tasca, \& G. De Carli, 2004. Scanning electron microscopy study of Tritrichomonas augusta. Parasitology Research, 94: 15861. DOI: $10.1007 / \mathrm{s} 00436-004-1189-2$

Bozner, P., 1996. The heat shock response and major heat shock proteins of Tritrichomonas mobilensis and Tritrichomonas augusta. Journal of Parasitology, 82: 103-111. DOI: $10.2307 / 3284124$
Brugerolle, G., 1973. Sur 1'existence de vrais kystes ches les trichomonadines intestinalis. Ultrastructure des kystes de Trichomitus batrachorum Perty 1852, Trichomitus sanguisugae Alexeie 1911, et Monocercomonas tipulae Mackinnon 1910. Comptes rendus de l'Académie des Sciences Paris Serie D, 277: 2193-2196. (In French)

Cepicka, I., V. Hampl, \& J. Kulda, 2010. Critical taxonomic revision of parabasalids with description of one new genus and three new species. Protist, 161: 400-433. DOI: 10.1016/j.protis.2009.11.005

Delvinquier, B.L.J. \& W.J. Freeland, 1988. Protozoan parasites of the cane toad, Bufo marinus, in Australia. Australian Journal of Zoology, 36: 301-316. DOI: 10.1071/ ZO9880301

Dimasuay, K.G.B., O.J.Y. Lavilla, \& W.L. Rivera, 2013. New hosts of Simplicimonas similis and Trichomitus batrachorum identified by $18 \mathrm{~S}$ ribosomal RNA gene sequences. Journal of Parasitology Research, 2013: 1-5. DOI: $10.1155 / 2013 / 831947$

Dimasuay, K.G.B. \& W.L. Rivera, 2013. Molecular characterization of trichomonads isolated from animal hosts in the Philippines. Veterinary Parasitology, 196: 289-295. DOI: $10.1016 /$ j.vetpar.2013.03.019

Dobell, C., 1909. Researches on the intestinal protozoa of frogs and toads. Journal of Cell Science, s2-53: 201-278.

Farmer, M., 1993. Ultrastructure of Ditrichomonas honigbergii N.G., N.Sp. (Parabasalia) and its relationship to amitochondrial protists. Journal of Eukaryotic Microbiology, 40: 619-626. DOI: 0.11111/j.15507408.1993.tb06119.x

Frank, W., 1984. Non-hemoparasitic protozoans. In: Hoff, G., F. Frye, \& E. Jacobson (eds), Diseases of amphibians and reptiles. Plenum Press, New York and London, pp. 296298. DOI: 10.1007978-1-4615-9391-1

Germot, A., G. Brugerolle, \& E. Viscogliosi, 1996. The undulating membrane of trichomonads - The structure and immunolabelling of its cytoskeleton. European Journal of Protistology, 32: 298-305. DOI: 10.1016/S0932-4739(96) 80052-6

Gillespie, S.H. \& R. Pearson, 2001. Principles and practice of clinical parasitology. John Wiley \& Sons Ltd, England, pp. 243-268. DOI: 10.1002/0470842504

Global Invasive Species Database, 2005. Rhinella marina (=Bufo marinus). http://issg.org/database/species/ ecology.asp?si $=113 \& \mathrm{fr}=1 \& \mathrm{sts}=\&$ lang $=\mathrm{EN} . \quad$ Accessed 7 April 2018.

Gookin, J.L., S.H. Stauffer, \& M.G. Levy, 2007. Identification of Pentatrichomonas hominis in feline fecal samples by polymerase chain reaction assay. Veterinary Parasitology, 
145: 11-15. DOI: 10.1016/j.vetpar.2006.10.020

Grabensteiner, E., I. Bilic, T. Kolbe, \& M. Hess, 2010. Molecular analysis of clonal trichomonad isolates indicate the existence of heterogenic species present in different birds and within the same host. Veterinary Parasitology, 172: 53-64. DOI: 10.1016/j.vetpar.2010.04.015

Granger, B.L., S.J. Warwood, M. Benchimol, \& W. De Souza, 2000. Transient invagination of flagella by Tritrichomonas foetus. Parasitology Research, 86: 699-709. DOI: 10.1007/ PL00008555

Hall, T.A., 1999. BioEdit: a user-friendly biological sequence alignment editor and analysis program for Windows 95/98/ NT. Nucelic Acids Symposium Series, 41: 95-98.

Honigberg, B., 1953. Structure, taxonomic status, and host list of Tritrichomonas batrachorum (Perty). Journal of Parasitology, 39: 191-208. DOI: 10.2307/3274117

Honigberg, B.M., W.A. Daniel, \& C. Mattern, 1972. Fine structure of Trichomitus batrachorum (Perty). Journal of Protozoology, 19: 446-453. DOI: 10.1111/j.15507408.1972.tb03499.x

Jensen, E.A. \& D.M. Hammond, 1964. A morphological study of Trichomonads and related flagellates from the bovine digestive tract. Journal of Protozoology, 11: 386-394. DOI: 10.1111/j.1550-7408.1964.tb01768.x

Kamaruddin, M., M. Tokoro, M.M. Rahman, S. Arayama, A.P.N. Hidayati, D. Syafruddin, P.B.S. Asih, H. Yoshikawa, \& E. Kawahara, 2014. Molecular characterization of various trichomonad species isolated from humans and related mammals in Indonesia. Korean Journal of Parasitology, 52: 471-478. DOI: 10.3347/ kjp.2014.52.5.471

Kim, Y.A., H.Y. Kim, S.H. Cho, H.I. Cheun, J.R. Yu, \& S.E. Lee, 2010. PCR detection and molecular characterization of Pentatrichomonas hominis from feces of dogs with diarrhea in the Republic of Korea. Korean Journal of Parasitology, 48: 9-13. DOI: $10.3347 / \mathrm{kjp} .2010 .48 .1 .9$

Kumar, S., G. Stecher, \& K. Tamura, 2016. MEGA7: Molecular Evolutionary Genetics Analysis. Molecular Biology and Evolution, 33(7): 1870-1874.

Li, W.C., M. Ying, P.T. Gong, J.H. Li, J. Yang, H. Li, \& X.C. Zhang, 2016. Pentatrichomonas hominis: prevalence and molecular characterization in humans, dogs, and monkeys in Northern China. Parasitology Research, 115: 569-574. DOI: $10.1007 / \mathrm{s} 00436-015-4773-8$

Lockwood, B.C., M.J. North, \& G.H. Coombs, 1984. Trichomonas vaginalis, Tritrichomonas foetus, and Trichomitus batrachorum: comparative proteolytic activity. Experimental Parasitology, 58: 245-253. DOI: 10.1016/0014-4894(84)90041-9
Malik, S.B., C.D. Brochu, I. Bilic, J. Yuan, M. Hess, J.M. Logsdon, \& J.M. Carlton, 2011. Phylogeny of parasitic parabasalia and free-living relatives inferred from conventional markers vs. Rpbl, a single-copy gene. PLoS One, 6: e20774. DOI: 10.1371/journal.pone.0020774

Marinkelle, C., 1968. Tritrichomonas batrachorum Perty from some Colombian toads. Mitteilungen aus dem Instituto ColomboAleman de Investigaciones Cientificas, 2: 29-31.

Mattern, C., B. Honigberg, \& W. Daniel, 1973. Fine-structural changes associated with pseudocyst formation in Trichomitus batrachorum. Journal of Protozoology, 20: 222 -229. DOI: 10.1111/j.1550-7408.1973.tb00869.x

Mostegl M.M., B. Richter, N. Nedorost, C. Lang, A. Maderner, N. Dinhopl, \& H. Weissenbock, 2012. First evidence of previously undescribed trichomonad species in the intestine of pigs? Veterinary Parasitology, 185: 86-90. DOI: 10.1016/j.vetpar.2011.10.029

Ong, V.A. \& W.L. Rivera, 2010. Prevalence of Trichomonas vaginalis in vaginal swabs from sex workers in Angeles City, Pampanga, Philippines as detected by PCR, Tropical Medicine and Health, 38: 29-34. DOI: 10.2149/tmh.200917

Pereira-Neves, A., K.C. Ribeiro, \& M. Benchimol, 2003. Pseudocysts in trichomonads--new insights. Protist, 154: 313-329. DOI: 10.1078/143446103322454095

Rivera, W.L., 2008. Phylogenetic analysis of Blastocystis isolates from animal and human hosts in the Philippines. Veterinary Parasitology, 156: 178-182. DOI: 10.1016/ j.vetpar.2008.06.001

Rivera, W.L., A.J.B. Lupisan, \& J.M.P. Baking, 2008. Ultrastructural study of a tetratrichomonad isolated from pig fecal samples. Parasitology Research, 103: 1311-1316. DOI: $10.1007 / \mathrm{s} 00436-008-1134-\mathrm{x}$

Samuels, R., 1957. Studies of Tritrichomonas batrachorum I. The trophic organism. Journal of Protozoology, 4: 110-118. DOI: 10.1111/j.1550-7408.1957.tb02494.x

Solís, F., R. Ibáñez, G. Hammerson, B. Hedges, A. Diesmos, M. Matsui, J.M. Hero, S. Richards, L. Coloma, S. Ron, E. La Marca, J. Hardy, R. Powell, F. Bolaños, G. Chaves, \& P. Ponce, 2009. Rhinella marina. IUCN Red List Threat Species. http://www.iucnredlist.org/details/41065/0. Accessed 7 August 2017.

Stoeck, T. \& S. Epstein, 2003. Novel eukaryotic lineages inferred from small-subunit rRNA analyses of oxygendepleted marine environments. Applied and Environmental Microbiology, 69: 2657-2663. DOI: 10.1128/ AEM.69.5.2657-2663.2003

Wartoń, A. \& B.M. Honigberg, 1979. Structure of trichomonads 
as revealed by scanning electron microscopy. Journal of Protozoology, 26: 56-62. DOI: 10.1111/j.15507408.1979.tb02732.x

Whittington, M.J., 1951. Observations upon a trichomonad (Protozoa: Mastigophora) from the gut of the snake, Vipera ammodytes L. Parasitology, 41: 274-281. DOI: 10.1017/ S0031182000084110 